\title{
BMJ Open Enhancing the public health impact of latent tuberculosis infection diagnosis and treatment (ACT4): protocol for a cluster randomised trial
}

\author{
Olivia Oxlade, ${ }^{1}$ Anete Trajman, ${ }^{1,2}$ Andrea Benedetti, ${ }^{1}$ Mênonli Adjobimey, ${ }^{3}$ \\ Victoria J Cook, ${ }^{4,5}$ Dina Fisher, ${ }^{6}$ Gregory James Fox, ${ }^{7}$ Federica Fregonese, ${ }^{1}$ \\ Panji Hadisoemarto, ${ }^{8}$ Philip C Hill, ${ }^{9}$ James Johnston, ${ }^{4,10}$ Richard Long, ${ }^{11}$ \\ Joseph Obeng, ${ }^{12}$ Rovina Ruslami, ${ }^{13}$ Chantal Valiquette, ${ }^{1}$ Dick Menzies ${ }^{1}$
}

To cite: Oxlade 0, Trajman A, Benedetti $A$, et al. Enhancing the public health impact of latent tuberculosis infection diagnosis and treatment (ACT4): protocol for a cluster randomised trial. BMJ Open 2019;9:e025831. doi:10.1136/ bmjopen-2018-025831

- Prepublication history for this paper is available online. To view these files, please visit the journal online (http://dx.doi. org/10.1136/bmjopen-2018025831).

Received 3 August 2018 Revised 17 December 2018 Accepted 24 January 2019

Check for updates

(C) Author(s) (or their employer(s)) 2019. Re-use permitted under CC BY-NC. No commercial re-use. See rights and permissions. Published by BMJ.

For numbered affiliations see end of article.

Correspondence to Dr Dick Menzies; dick.menzies@mcgill.ca

\section{ABSTRACT}

Introduction Treatment of latent tuberculosis (TB) infection (LTBI) is an important component of the EndTB strategy. However, the number of individuals who successfully complete LTBI treatment remains low as there are losses at all steps in the LTBI 'cascade-of-care'. The reasons for these losses are variable and highly dependent on the setting. We have planned a trial of a standardised public health approach to strengthen the management of household contacts (HHCs) of newly diagnosed patients with pulmonary TB. Assessing costs related to approach is a secondary objective of the study.

Methods and analysis A cluster randomised trial will be conducted in 24 randomisation units (health facilities or groups of health facilities) in five countries. In Phase 1 , at intervention sites, we will conduct a standardised assessment of the current LTBI programme, with a focus on cascade-of-care endpoints. Standardised open-ended questionnaires on practices, knowledge, attitudes and beliefs regarding TB prevention are then administered to key patient groups and healthcare workers. At each site, local stake-holders will review study findings and select solutions based on their acceptability, cost and effectiveness. In Phase 2, intervention clinics will implement the selected solutions, along with contact measurement registries and regular in-service LTBI management training. Control sites will continue their usual LTBI care with no explicit evaluation, strengthening or training activities. The primary study outcome is the number of HHC initiating LTBI treatment per newly diagnosed active TB patient, within 3 months of diagnosis of the index patient. An intention-to-treat analysis will be performed, using a Poisson regression approach. Ethics and dissemination Ethics approval from the MUHC ethical review board (ERB) was obtained in November 2015. During the study standardised tools will be developed and made publicly available. Key study findings and novel methodologic contributions will be detailed in publications and other dissemination activities.

Trial registration number NCT 02810678; Pre-Results.

\section{Strengths and limitations of this study}

- The study uses a standardised approach to tackle a complex problem- of latent tuberculosis (TB) diagnosis investigation and treatment- through a cascade-of-care approach and a locally driven evaluation and decision-making process to decide on solutions and strengthening activities directed at the latent TB infection (LTBI) programme.

- The study design combines the principles of randomisation with the practical reality that public health problems have multiple causes, and hence may need site-specific solutions.

- The small number of randomization units (health facilities) in our cluster randomised trial will limit power to detect smaller effects of the intervention.

- Components of the LTBI programme strengthening exercise will vary locally, making it difficult to judge the specific contribution of each solution implemented.

- Tools created as part of the strengthening process include a registry-based system for periodic evaluation of the LTBI cascade-of-care that corresponds to WHO TB reporting criteria.

\section{INTRODUCTION}

Tuberculosis (TB) is a leading cause of death worldwide. ${ }^{1}$ It is estimated that almost 2 billion people globally are infected with Mycobacterium tuberculosis. ${ }^{2}$ Modelling studies have projected that even with the implementation of new diagnostic and treatment tools, the goal of TB elimination by $2050^{3}$ will not be achieved without the treatment of persons with latent TB infection (LTBI), the reservoir for new TB cases. ${ }^{4}$

The WHO has recently endorsed treatment for LTBI as an important TB control strategy. ${ }^{5}$ Treatment for LTBI is known to be highly effective under programmatic conditions, ${ }^{6-8}$ however for treatment to be effective, 
individuals who are most at risk of developing TB need to be identified, screened and offered treatment. People who live with infectious TB patients (household contacts; HHC) are at especially high risk of becoming infected with $\mathrm{TB}$ and subsequently progressing to $\mathrm{TB}$ disease. Once individuals initiate treatment for LTBI, $61 \%$ complete treatment for LTBI, ${ }^{9}$ however contacts of newly diagnosed TB patients may be lost prior to initiating treatment for a variety of reasons, including: failure to identify those who should be screened; incomplete assessment and subsequent medical evaluation; providers failing to recommend preventive therapy to eligible contacts; and contacts refusing to accept treatment. Cumulatively, all of the losses that occur in the cascade prior to treatment initiation result in a greater loss than the proportion lost once treatment has been initiated. ${ }^{910}$

As most individuals with LTBI are asymptomatic, and so do not spontaneously seek medical attention, health systems must implement contact investigation programmes to identify and screen exposed contacts. In several published evaluations of large-scale LTBI treatment programmes, dropouts and losses have been documented at every stage of the LTBI cascade-of-care, in both high $^{11-14}$ and low-income countries. ${ }^{15} 16$

Although the types of health system bottlenecks and patients' barriers in the LTBI cascade are well established, ${ }^{9}$ the specific problems vary between and within countries. This means that site-specific evaluations are important to understand which problems lead to important losses during the cascade-of-care, ${ }^{17}$ and what local solutions may be relevant and effective.

We will conduct a pragmatic, cluster randomised controlled trial (ACT4) over 20 months, to evaluate the effectiveness of a standardised public health intervention.

The primary objective will be to estimate the increase in the number of HHCs initiating LTBI treatment per newly diagnosed index patient, within 3 months of diagnosis of the index patient. The secondary study objective is to assess the costs and cost-effectiveness of the LTBI programme evaluation and strengthening approach. In preparation for this trial, a pilot study was conducted to assess study feasibility and refine study tools in 12 health facilities in Brazil. Results from the Brazil pilot will be published as a separate manuscript.

\section{METHODS AND ANALYSIS \\ Study design}

This is a pragmatic cluster-randomised controlled trial in 24 randomisation units (health facilities or groups of health facilities) in Benin, Canada, Ghana, Indonesia and Vietnam. Detailed information about study sites can be obtained from the authors at the coordinating centre. Health facilities randomised to the intervention group will receive a 20 month public health intervention. The intervention consists of a first phase of standardised LTBI programme evaluation, followed by a process of stake-holder consultation and local selection of solutions (transition phase) followed by programme strengthening through implementation of these solutions (phase 2) (see figure 1). This is achieved through structured initial and in-service training and re-evaluation of progress using simple analytic tools based on LTBI management registries. During this time, control sites will continue programmes as usual with no explicit LTBI strengthening related activities.

\section{Study population, subsites and eligibility criteria}

The trial will include four health facilities in Canada (Calgary, Edmonton, Montreal and Vancouver) a low TB incidence country, plus 20 health facilities (or groups of health facilities) in four countries with intermediate to high TB incidence rates: Benin, Ghana, Indonesia and Vietnam. In 2017, WHO estimated annual incidence rates were respectively 59, 156, 391 and 133 per 100000 population. ${ }^{1}$

\section{Randomisation}

Randomisation of health facilities will take place before the start of phase 1 . The randomisation sequence will be stratified by country and restricted to ensure an approximate balance of annual number of patients with newly diagnosed pulmonary TB randomised to each arm within each country. Because the number of TB patients is not the same at all sites within each country, and between countries, a total of 1000 randomisation sequences will be generated using a computer programme developed by the study biostatistician. These 1000 sequences will be examined to verify if the number of expected index cases (and hence the expected number of HHCs) are approximately equal ( $48 \%-52 \%$ balance) overall in the two arms. Randomisations that result in reasonable balance will be selected, and in a final step, one of these sequences will be randomly selected from this subgroup of balanced randomization sequences. The study biostatician will not inform the coordinating centre of the randomization sequence until the start of phase 1 . The coordinating centre will then notify sites as to which health facilities are randomised to intervention or control.

\section{Blinding and minimisation of bias}

As the study is a cluster randomised trial, and the unit of randomisation is the health facility, it will not be possible to blind health facilities staff, patients with TB disease and their HHCs to their study allocation. In order to minimise bias, the study biostatician will be blinded to study arm throughout any analysis of study outcomes. We will limit contamination at control sites by collecting outcome data from pre-existing sources and minimising interaction of research staff with health facility staff.

\section{Outcomes}

The primary outcome will be the number of HHCs initiating treatment per newly diagnosed TB index patient within 3-4 months from index patient diagnosis (see detailed explanation below). A new TB index patient will be microbiologically confirmed using AFB smear, 
PHASE 1

Month 1-6

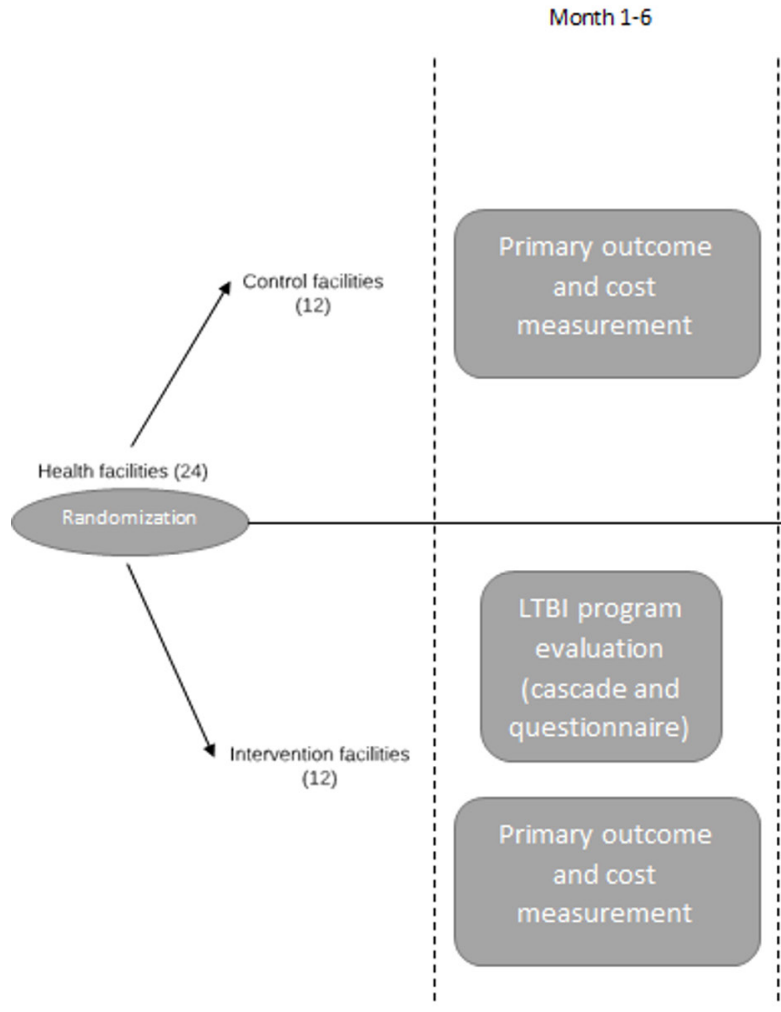

TRANSITION PHASE

PHASE 2

Month 11-20

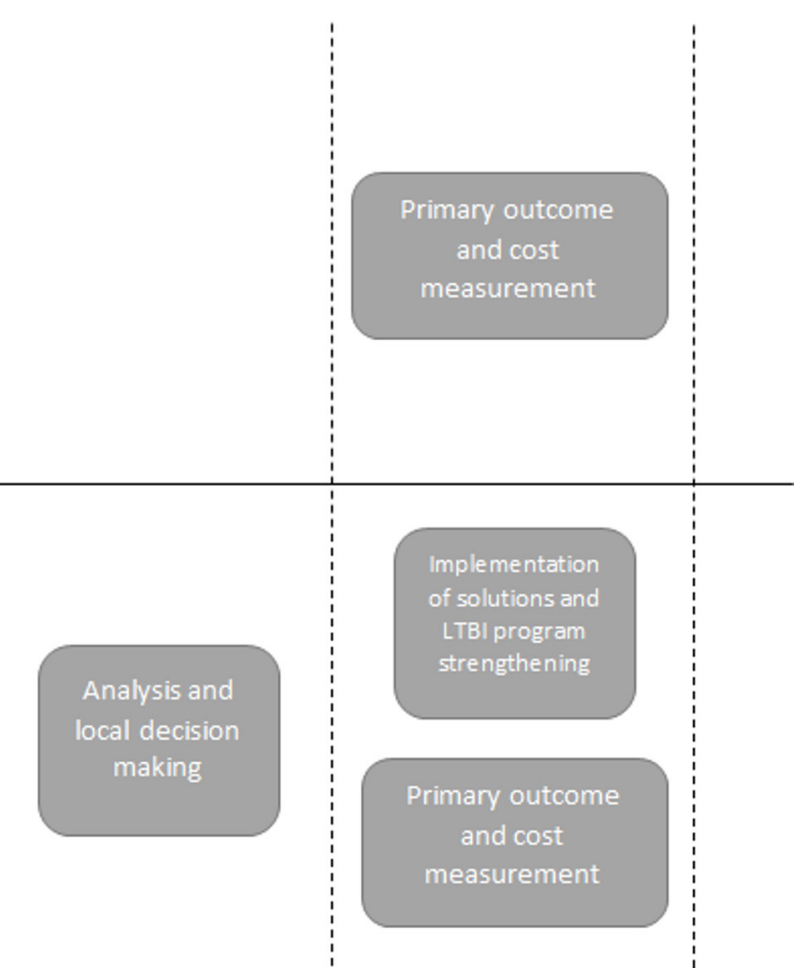

Figure 1 Schematic of study design.

culture, and/or molecular tests such as Xpert MTB/RIF, depending on local protocols. A HHC will be defined as someone who slept in the same house at least one night per week, or spent more than 1 hour in the house at least 5 days per week, on average, over the preceding 3 months. The house will be defined as the dwelling, or buildings, which the family unit occupies and uses regularly. In all sites treatment initiation for HHCs will be defined as clinical or pharmacy records indicating that a prescription has been issued or, in clinics were medications are given directly to patients, a HHCs being issued LTBI medication by a healthcare worker.

\section{Activities and data gathering in all health facilities}

Primary study outcome: the primary study outcome will be recorded at all intervention and control health facilities for the full duration of phase 1 (6 months) and for the last 6 months of phase 2, using a standardised form that is available from the coordinating centre. In each of the 6 month periods, the total number of index patients, the number of their contacts who were recorded in clinic documents, and the number of these HHCs who initiate LTBI therapy will be collected from existing clinical records, in both the control and intervention arms. Although the data sources will vary by site, the procedures to measure these outcomes will continue unchanged at intervention and control health facilities throughout the study. We will include all HHCs, of all ages that were identified, for new TB index patients diagnosed within the two 6 month intervals. For TB index patients diagnosed towards the end of each 6 month period in most sites we will allow up to three additional months for the HHCs to be started on LTBI treatment. In the Canadian sites 4 months will be allowed as HHCs tracing involves a baseline and 8-week post-exposure assessment prior to treatment initiation. HHCs started on LTBI treatment more than 3 months (or four in Canadian sites) after the diagnosis of the last index TB patient will not be considered to have achieved the primary study outcome.

Trial implementation costs and LTBI related health system costs: throughout the trial, costs related to the implementation of the LTBI programme evaluation and strengthening will be measured in each setting, using time and activity logs for research staff, investigators, healthcare workers and management staff involved in implementation. Country specific budgets will be used to obtain expenditures related to services, supplies and materials. To estimate LTBI related health system personnel costs, time and motion studies ${ }^{18}$ will be conducted at the start of phase 1 and the end of phase 2. Other information will be obtained at the level of the health facility using a costing questionnaire.

Phase 1: activities and data gathering in intervention health facilities

Evaluation of the LTBI programme at baseline using a cascade-of-care analysis: in phase 1 , a standardised LTBI programme evaluation will be conducted at each 


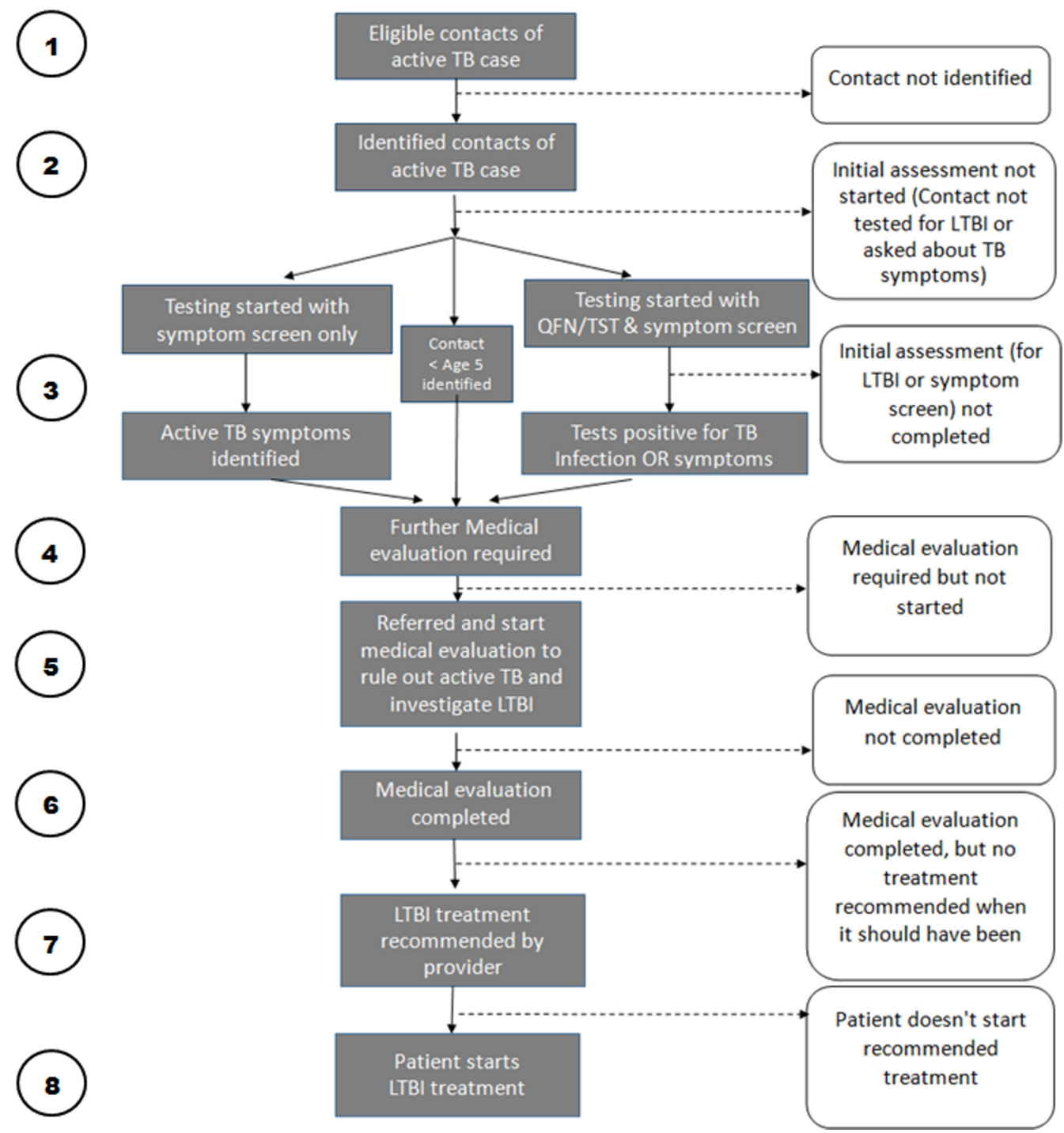

Figure 2 LTBI cascade-of-care framework, showing how losses can occur at each step. LTBI, latent tuberculosis infection; QFN, Quantiferon; TB, tuberculosis.

intervention health facility to identify local barriers to LTBI diagnosis and treatment initiation, from the healthcare workers and users' perspective. This will inform the selection of solutions applied in phase 2 . Two tools will be used: (1) the LTBI cascade-of-care analysis and (2) interviewer-administered questionnaires.

a. LTBI cascade-of-care analysis: existing data sources will be used - including registries of contact investigations, tuberculin testing or LTBI treatment, or individual medical records including but not limited to treatment cards of TB patients on which their contacts are listed. The eight steps outlined in the schematic (figure 2) will be used as a framework for cascade-of-care data collection. Key steps in the framework are as follows: (i) the number index TB patients, (ii) the number of their HHCs who are identified as eligible for symptom screening and LTBI testing; (iii) the number of
HHCs who are questioned about symptoms only, and the number who complete LTBI testing; (iv) the number of those tested who are positive; (v) the number of contacts with positive LTBI tests who have a medical evaluation, (vi) the number of HHCs with positive LTBI tests who complete medical evaluation, (vii) the number of contacts that were recommended to initiate treatment for LTBI and (viii) the number of patients who ultimately accept the recommendation to start therapy. See figure 2 for more details.

b. Interviewer-administered questionnaires: these standardised open-ended questionnaires will be used to assess practices, knowledge, attitudes and beliefs regarding the key components of the LTBI management system, including TB prevention, management of contacts, experiences with LTBI diagnosis and therapy, as well as TB-related knowledge, attitudes and beliefs. 
Four groups will be interviewed; (i) index TB patients (ii) HHCs of index TB patients, (iii) parents of young ( $<5$ years) child-aged HHCs of index TB patients and (iv) healthcare workers). At each intervention health facility approximately 10-20 participants from each of these groups will be asked to consent to participating in the study. Data will be directly entered into a secure online data storage platform.

Transition from phase 1 to phase 2: analysis of phase 1 results, selection of solutions and preparation for phase 2

Data from the LTBI cascade-of-care evaluation will be used to identify which steps account for the greatest losses in the LTBI cascade. Information from the questionnaires will be used to identify why these losses might be occurring. Potential solutions will be identified and may apply to different points in the LTBI cascade and could include: healthcare worker education, ${ }^{19}{ }^{20}$ home visits by healthcare workers ${ }^{21}$ incentives provided to patients to return for test results. ${ }^{22}$ Estimates of the possible magnitude of the effect of these different solutions will be derived from a systematic review and meta-analysis of relevant studies. Health system costs related to running the current LTBI programme at baseline, and costs of potential solutions will be collected in each country. The potential cost-effectiveness of implementing different solutions to improve the cascade will be evaluated using a discrete event simulation model ${ }^{23}$ that reflects the cascade-of-care of LTBI care framework (figure 3).

This information will be shared with stakeholders in each country and intervention health facility during a series of meetings which will have the goal of selecting the most appropriate solution(s). Criteria for selection of solutions will include: evidence of efficacy from published studies, anticipated feasibility and sustainability at participating health facilities, acceptability by healthcare workers and patients, and affordability. Site specific solutions will be reviewed with key programme staff. The specific preparation required will depend on the solutions chosen. As part of the planning for phase 2 it is essential to establish what 'strengthened LTBI management' means in each site. Points to consider in the planning process include ensuring health care workers (HCW) have sufficient training in LTBI related topics. Meetings will be arranged with key stakeholders

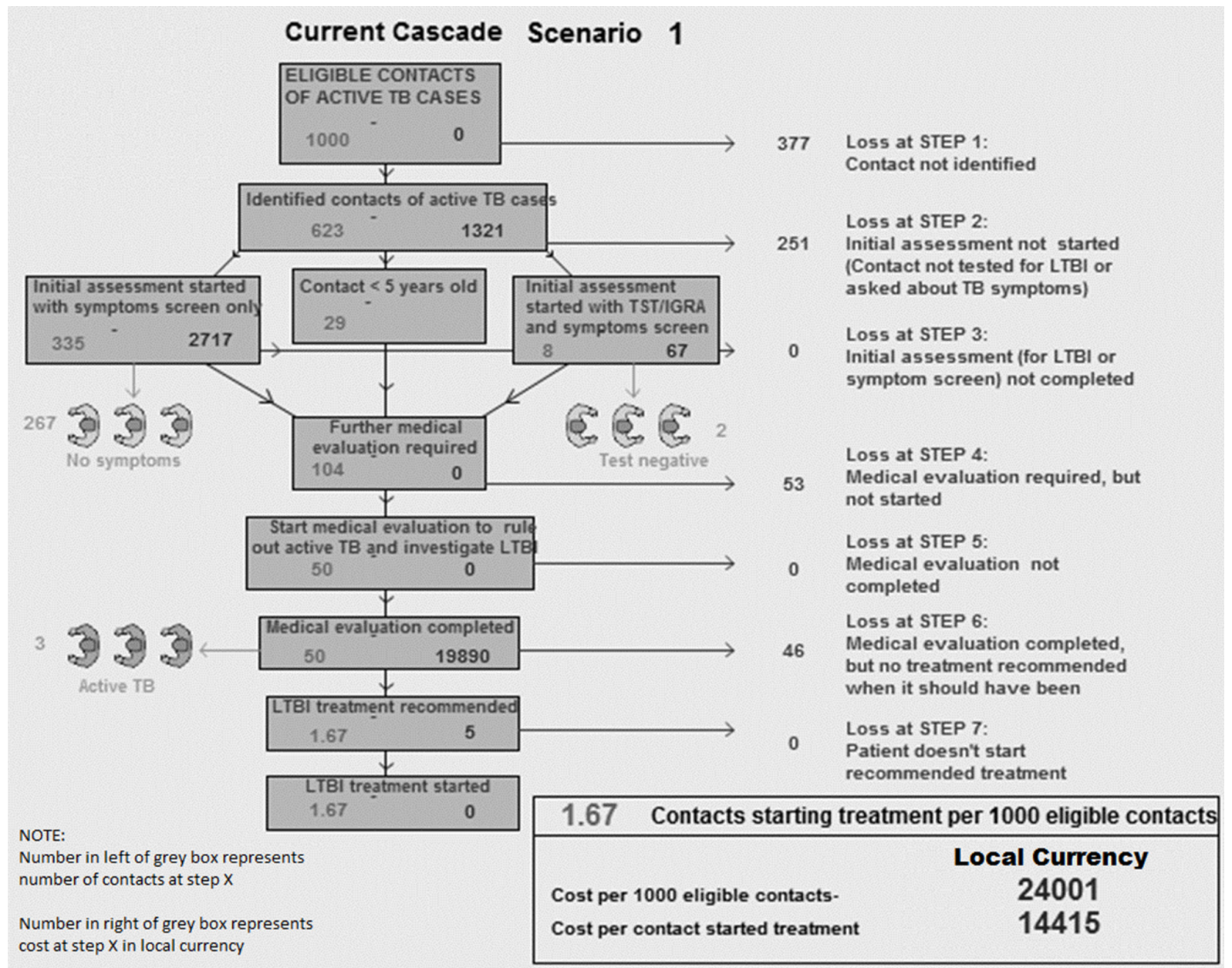

Figure 3 The eight-step LTBI cascade-of-care framework for country X, adapted as a discrete event simulation model used to estimate at baseline, the number of individuals initiating LTBI treatment and the cost per 1000 contacts initiating treatment. Losses between steps are indicated on the right-hand side of the figure. IGRA, Interferon Gamma Release Assay; LTBI, latent tuberculosis infection; TB, tuberculosis. 
to confirm that they are in agreement with the project, understand the trainings that are essential to the success of strengthening, and the importance of not 'contaminating' control sites with study related material.

\section{Phase 2: activities and data gathering in intervention health} facilities

LTBI Programme Strengthening and Implementation of Solutions:

a. Clinical training: at the start of phase 2, clinical training should be carried out for different types of HCW (doctors, nurses, nurse aids, mid-level and auxiliary personnel). Topics will include: how to identify HHCs and conduct a contact investigation, initial assessment of contacts, medical evaluation and LTBI diagnosis (including how to administer and read Tuberculin Skin Test (TST)). Procedures for medical evaluation- to exclude active TB and to decide who should receive latent TB treatment will also be included. Treatment for LTBI will be discussed, although the choice of the LTBI regimen is entirely up to the TB programme. Material to use in sessions will be developed as part of the study. Initial training sessions involving key staff can be organised to 'train the trainer', with subsequent sessions led by those trained to larger groups of HCW. Clinical materials (including TST material and supplies) will also need to be available at each health facility. Additional educational materials for patients can be distributed as needed.

A specific registry for the management of contacts with LTBI will be developed. The registry will support data collection and management of HHCs who progress through the cascade, using an index case cohort approach. An important part of the training will be to review how to use the new registry - for clinical management, and to assess progress in LTBI management (ie, repeat cascade analysis, see below). The registry will help reinforce the steps in contact investigation and LTBI management.

b. Implementation of solutions. Once the initial clinical training has been completed implementation of the selected solutions can occur. This includes if the solutions are to be implemented outside of the clinic (such as a media campaign, or a community event).

c. In-service training. Within 1 week of the initial training, research staff will visit each intervention health facility to review all steps in contact investigation and LTBI diagnosis and treatment. Clinic staff will be asked to perform TST on contacts two to 3 days before each scheduled in-service training visit by research staff. During these in-service training visits, the research staff will read the TST results together with the clinic staff, thereby providing reinforcement of teaching, and quality control of initial TST readings.

In-service training visits will be conducted weekly for the first month and then reduced to twice a month for the next 2 months. After 3 months, if the programme is working well, in-service training visits can be reduced to once a month.

d. Repeat cascade analysis: once the registries are being filled out correctly, they can be used periodically to re-evaluate the LTBI cascade. This will be done during the in-service training visits 3 months after initial training is completed. If new problems are identified from the repeat cascade analysis, they can immediately be discussed with clinic staff to identify appropriate corrective actions.

Detailed standard operating procedures (SOPs) and forms used for all data gathering activities and other study activities described above can be obtained from study authors at the coordinating centre. Details on data management and storage are also included in SOPs.

\section{Activities in control health facilities}

The only study related activities in the control health facilities throughout the 20 months study will be the collection of study outcomes and health system cost data. This will be done for the same time periods as in intervention facilities (figure 1). Outcome data will be collected from pre-existing sources in order to minimise the interaction with health facility staff at control sites (see next section on Contamination for more detail). All other TB and LTBI related activities that were in place at the start of the study will continue as usual, including any new interventions or training introduced at all facilities by the TB programme. Tuberculin material (PPD) and isoniazid will be provided to sites if needed.

\section{Sample size}

Twenty-four randomisation units (single health facilities or groups of health facilities) were identified, such that each unit anticipated seeing 20 index TB patients and 80 HHCs over a 6 month period. The anticipated number of TB patients and HHCs in each country is shown in table 1. The annual number of TB patients was obtained from pre-existing registries at each of the sites.

For each randomisation unit, the number of HHCs expected to initiate treatment per index TB patient was generated in a simulation exercise, using a Poisson distribution with a rate that depended on the effect of the intervention, the effect of time and a normally distributed random effect for each randomisation unit. We varied the potential effect of the intervention from $5,10,15,30$ contacts initiating treatment per 100 index TB patients. The variance of the random effect was set at $0.7,1.4$ and 2.8. These variances resulted in approximate ICCs (intraclass correlation coefficients) of $0.16-0.40$. We generated 500 datasets for each distinct combination of data generation parameters (12 scenarios).

Using this approach we estimated power as the number of generated datasets that resulted in a statistically significant value. As shown in table 2, we will have $73 \%-75 \%$ power to detect a statistically significant (alpha $=0.05)$ effect of the intervention if the increase in 
Table 1 Anticipated number of index tuberculosis patients and their household contacts at participating sites

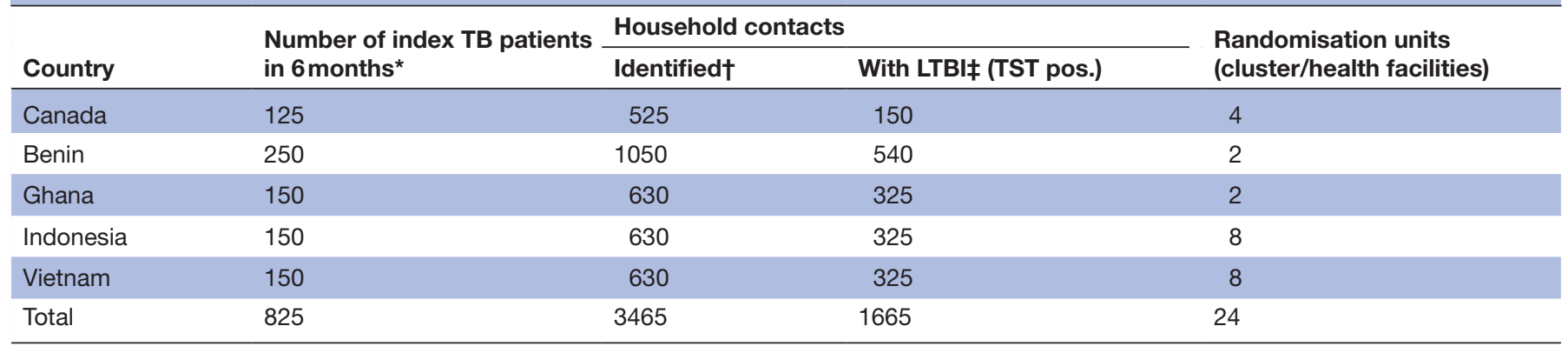

${ }^{*}$ Approximate number based on retrospective information provided from TB registries at sites.

†Estimated based on 4.2 contacts identified per active TB case - from systematic review. ${ }^{33}$

†Estimated based on prevalence of LTBI among contacts screened: of $51.5 \%$ in low-income and middle-income countries (LMIC) ${ }^{28} 33$ and $28.1 \%$ in Canada. ${ }^{28}$

LTBI, latent tuberculosis infection; TB, tuberculosis.

the number of HHCs initiating LTBI treatment (between phases 1 and 2), is at least 10 per 100 index TB patients. However, we will have more than $96 \%$ power to detect a significant effect of the intervention, if it results in at least 15 more HHCs initiating treatment per 100 index TB patient.

\section{Data analysis}

Primary analysis

An intention-to-treat analysis will be performed, using a Poisson regression approach. We will use a marginal Poisson regression model with identity link (see Breslow ${ }^{24}$ for specification), estimated via generalised estimating equations, and using robust standard errors, to account for clustering at the level of the randomization unit.

Table 2 Power with 24 randomisation units for various Intra-class correlations coefficient in all countries, with alpha $=0.05$

\begin{tabular}{|c|c|}
\hline $\begin{array}{l}\text { Difference in the change } \\
\text { from phase } 1 \text { to phase } \\
2 \text { in the number of } \\
\text { household contact } \\
\text { starting latent tuberculosis } \\
\text { infection treatment per } \\
\text { index tuberculosis patient } \\
\text { between the intervention } \\
\text { and control arms }\end{array}$ & $\begin{array}{l}\text { Power } \\
\text { (\%) }\end{array}$ \\
\hline 5 & 28 \\
\hline 5 & 32 \\
\hline 5 & 26 \\
\hline 10 & 74 \\
\hline 10 & 76 \\
\hline 10 & 73 \\
\hline 15 & 96 \\
\hline 15 & 97 \\
\hline 15 & 97 \\
\hline 30 & 100 \\
\hline 30 & 100 \\
\hline 30 & 100 \\
\hline
\end{tabular}

Because the number of randomization units is less than 40 , we will use a correction for few clusters. ${ }^{25}$ Using an identity link will allow us to describe the effect of the intervention in terms of a rate difference.

The dependent variable will be the number of HHCs who initiated treatment for LTBI per index TB patient. Two time points will be included - Phase 1: the 6 months before the programme strengthening begins, and Phase 2: during the last 6 months, after programme strengthening has been implemented. The model will include terms for the intervention, study phase, and the interaction between study phase and intervention. The primary focus of the analysis will be the interaction term, which is interpreted as the difference in the change from phase 1 to phase 2 in the number of HHCs starting LTBI treatment per index TB patient between the intervention and control arms (ie, a difference of differences).

When using the identity link, it is possible that the model predicts values $<0$. If our model predictions are not greater than 0 or in the case of convergence problems, we will consider (i) a Poisson regression model with fixed intercepts for each site ${ }^{26}$ or (ii) using a log link and $\log (\mathrm{TBi})$ as the offset. Overdispersion will be investigated and accounted for if necessary. ${ }^{27}$

In secondary analyses, we will adjust the model for level of economic development at the country level. We will also stratify findings by low-income and middle-income countries (LMIC) and Canadian sites in order to adjust for potential between country differences such as treatment duration and estimate the effect of the intervention separately in each country. In additional analyses, using methods described above, we will compare the outcomes in phase 1 to phase 2, separately in the intervention and control arms.

\section{Economic analysis}

For the secondary objective, resource use related to the implementation of the ACT4 approach and the downstream increases in LTBI-related clinical care will be estimated. This will be essential to inform those considering implementation of a similar LTBI evaluation and 
strengthening package in other settings and the scale up of the approach. We will consider all resources related to the programme evaluation and strengthening over the full duration of the study. The perspective of the evaluation will be from the health system. Information on costs in the following categories will be based on: (1) material, services and supplies -central and site expenditures, as well as health facility budgets, (2) in-country research personnel -site budgets, salary scales and TAM studies to estimate time spent on relevant activities, (3) Montreal Coordinating Centre personnel time spent on various activities, and central research budgets; (4) in-country TB management personnel-in-depth interviews to create a narrative of time spent on relevant activities, (5) health centre management personnel-based on a similar narrative and (6) health centre clinical personnel - TAM studies and health facility salary scales. Salary scales for management and clinic staff will be used to estimate personnel costs based on their time. Additional costs related to LTBI clinical care will be estimated using an ingredients based approach to calculate per visit costs at each step of the cascade, together with information on the number of contacts being seen at different cascade steps at each site.

Incremental cost-effectiveness ratios will be used to assess the cost-effectiveness of the LTBI evaluation and strengthening package versus the status quo. Sensitivity analyses will be conducted to examine the robustness of the findings. We will also consider the impact of varying key cost parameters such as salaries for HCW and TB management personnel (using reported ranges) and personnel time devoted to LTBI programme strengthening for different categories of staff.

\section{Study monitoring}

A data monitoring committee is not required as no safety issues are anticipated in a public health evaluation trial. Research staff from the coordinating centre will visit all study sites every 6 months in order to review study procedures and progress. Investigator meetings will be held annually to review study progress and review future plans.

\section{Roles and responsibilities}

The trial will be managed by a coordinating centre who are based in Montreal. Research staff at the coordinating centre will be responsible for leading development of study material and tools, writing SOPs and assisting sites with data analysis. A Scientific Advisory Committee has been established to ensure scientific oversight.

\section{Patient and public involvement}

The research question considered in this trial is oriented towards improving patient outcomes and experiences throughout the LTBI cascade or care. Patients were not specifically included in the study development and design however they are integral to its execution. The study is designed to incorporate information from patients with active $\mathrm{TB}$, their contacts and parents of child contacts, by interviewing them in order to learn about their barriers and needs. This information is used to select solutions that are implemented in phase 2 of the study. Study results will be disseminated to participating clinics who may choose to share information with patients and study participants. The intervention was not assessed by patients themselves prior to initiation of the trial.

\section{Study schedule and trial status}

The first site began phase one on 1 August 2016, and the last site will complete phase 2 in December of 2018 (final outcome data collection in March 2019). Once all sites have completed outcome data collection for phase 2, data analysis will begin.

\section{Ethics and dissemination \\ Ethics}

Ethics approval was first obtained from the McGill University Health Centre ethical review board. The study began in each participating site after local ethics approval. Any protocol amendments should be circulated to sites once approved by the coordinating centres ERB. They must then be approved locally at each site.

For phase 1 of the study, individual informed consent will be obtained for the questionnaires and confidentiality will be ensured. For the cascade indicators and or study outcomes, consent is not required because data are aggregated and anonymously collected in each clinic. Likewise, for phase 2 informed consent will not be required for any individuals. Instead, public health administrators and TB control managers' approval will be sought for use of aggregate health facility data.

\section{Dissemination}

During the study standardised tools will be developed. These tools will be made publicly available through sites such the Find TB Resources website (http://www.findtbresources. cdc.gov). Trial results will be disseminated within each country as the investigators in all countries have close links to the leadership of their national TB programmes. The Contact Management Registries and reporting procedures are designed for easy uptake by NTP programmes and staff. Study findings and novel methodological contributions will be detailed in scientific manuscripts for publication. Data generated from the study will be made publicly available by request from the coordinating centre, 1 year after publication of the main results.

\section{DISCUSSION}

Many studies have considered the importance of contact investigation for $\mathrm{HHCs}^{28}$ and assessed methods to improve treatment completion for TB and LTBI, ${ }^{29}{ }^{30}$ but none have focused on strengthening the entire cascade of LTBI care. This protocol describes a unique public health intervention that aims to strengthen an LTBI programme according to its specific needs. The approach is standardised yet can be integrated into any setting and any stage of programme strengthening. This type of public health approach has been used 
successfully in other settings. In Senegal and Mali, hospital staff were trained to conduct maternal mortality review, which in turn reduced maternal mortality. ${ }^{31}$ In Nepal, the facilitation of women's group meetings was shown to improve birth outcomes. ${ }^{32}$ In both studies, the intervention was a structured evaluation; the actual changes that improved outcomes were site-specific.

Treatment initiation was selected as the trial primary end-point as the majority of loss in the LTBI cascade occurs before individuals begin treatment ${ }^{9}$ and improving treatment adherence has already been the focus of many research studies. ${ }^{28}{ }^{29}$ Treatment initiation is an outcome that can be evaluated much more quickly than treatment completion which fits better with the approach used in the trial of ongoing adjustments in real-time as part of a cycle of evaluation.

If the approach is found to be both successful and cost-effective, optimising the approach and ensuring that is sustainable will be essential. To assess its sustainability and refine some of the more complex tools used in the main study, we plan to provide the intervention to control sites after the study is complete. This add-on also minimises the ethical difficulties of withholding the interventions from one group - as eventually all groups will have the potential benefits of the trial.

The launch of this study is timely as it coincides with calls from the WHO to expand LTBI programmes in LMIC countries. ${ }^{5}$ As part of our study we will tailor tools to make them harmonise with WHO policy. For example, the LTBI registry used will incorporate LTBI indicators adopted by the WHO. We believe the results of this study will help low and middle-income countries prepare for the major shift in TB prevention that lies ahead.

\section{Author affiliations}

${ }^{1}$ McGill International TB Centre, McGill University, Montreal, Quebec, Canada

${ }^{2}$ Internal Medicine Graduate Program, Federal University of Rio de Janeiro, Rio de Janeiro, Brazil

${ }^{3}$ Centre National Hospitalier, Universitaire de Pneumo-Pthisiologie de Cotonou, Cotonou, Benin

${ }^{4} \mathrm{BC}$ Centre for Disease Control, Vancouver, British Columbia, Canada

${ }^{5}$ Department of Medicine, University of British Columbia, Vancouver, British

Columbia, Canada

${ }^{6}$ University of Calgary, Calgary, Alberta, Canada

${ }^{7}$ Faculty of Medicine and Health, The University of Sydney, Sydney, Australia

${ }^{8}$ Department of Public Health, Faculty of Medicine, TB-HIV Research Center,

Universitas Padjadjaran, Bandung, Indonesia

${ }^{9}$ Faculty of Medicine, Centre for International Health, University of Otago, Otago, New Zealand

${ }^{10}$ Division of Respiratory Medicine, Department of Medicine, University of British Columbia, Vancouver, British Columbia, Canada

${ }^{11}$ Faculty of Medicine and Dentistry, University of Alberta, Edmonton, Alberta,

Canada

${ }^{12}$ Ministry of Health, Accra, Ghana

${ }^{13}$ Department of Biomedical Sciences, Faculty of Medicine, TB-HIV Research Center, Universitas Padjadjaran, Bandung, Indonesia

Contributors DM wrote the proposal for this study that was awarded funding. AT, $\mathrm{PCH}, \mathrm{AB}$ and 00 made important intellectual contributions to the study protocol. All authors (00, AT, AB, MA, VJC, DF, GJF, FF, PH, PCH, JJ, RL, JO, RR, CV and DM) contributed to multiple revisions of the study protocol and study instruments. $A B$ and DM performed the statistical calculations for sample size and study power. 00 wrote the first draft of the manuscript. $A B, A T, D M$,and $P C H$ contributed substantially to the manuscript versions. All authors (00, AT, AB, MA, VJC, DF, GJF, FF, PH, PCH, $\mathrm{JJ}, \mathrm{RL}, \mathrm{JO}, \mathrm{RR}, \mathrm{CV}$ and DM) revised and approved the final manuscript version. The study sponsor played no role in any aspect of the study.

Funding This work was supported by the Canadian Institute of Health Research (Contact information: 160 Elgin Street, 9th Floor, Address Locator 4809A, Ottawa ON K1A 0W9, Canada) Grant number FDN- 143350.

Competing interests None declared.

Patient consent for publication Not required.

Ethics approval Ethics approval from the coordinating centre was obtained from the McGill University Health Centre ethical review board (ERB) on 27 November 2015 (15-291-MUHC).

Provenance and peer review Not commissioned; externally peer reviewed.

Open access This is an open access article distributed in accordance with the Creative Commons Attribution Non Commercial (CC BY-NC 4.0) license, which permits others to distribute, remix, adapt, build upon this work non-commercially, and license their derivative works on different terms, provided the original work is properly cited, appropriate credit is given, any changes made indicated, and the use is non-commercial. See: http://creativecommons.org/licenses/by-nc/4.0/.

\section{REFERENCES}

1. Global Tuberculosis Report 2017. World Health Organization, 2017. http://www.who.int/tb/publications/global_report/gtbr2017_main_ text.pdf?ua=1 (accessed 2 Aug 2018).

2. Houben RM, Dodd PJ. The global burden of latent tuberculosis infection: a re-estimation using mathematical modelling. PLoS Med 2016;13:e1002152.

3. Sustainable Development Goals. United Nations of Sustainable Development. United Nations, 2018. http://www.un.org/sustainabled evelopment/sustainable-development-goals/ (accessed 2 Aug 2018)

4. Uplekar M, Weil D, Lonnroth K, et al. WHO's new end TB strategy. Lancet 2015;385:1799-801.

5. Guidelines on the Management of Latent Tuberculosis Infection. World Health Organization, 2015. http://apps.who.int/iris/ bitstream/handle/10665/136471/9789241548908_eng.pdf jsessionid=0FAF6CBF844C75B7770C124D7A055599? sequence $=1$ (accessed 2 Aug 2018).

6. Hopewell PC, Fair E, Miller C. Recommendations for investigating contacts of persons with infectious tuberculosis in low- and middleincome countries. World Health Organization 2012 http://apps. who. int/iris/bitstream/10665/77741/1/9789241504492_eng.pdf (accessed 2 Aug 2018).

7. Bucher HC, Griffith LE, Guyatt GH, et al. Isoniazid prophylaxis for tuberculosis in HIV infection: a meta-analysis of randomized controlled trials. AIDS 1999;13:501-7.

8. Smieja MJ, Marchetti CA, Cook DJ, et al. Isoniazid for preventing tuberculosis in non-HIV infected persons. Cochrane Database Syst Rev 2000:CD001363.

9. Alsdurf $\mathrm{H}$, Hill PC, Matteelli A, et al. The cascade of care in diagnosis and treatment of latent tuberculosis infection: a systematic review and meta-analysis. Lancet Infect Dis 2016;16:1269-78.

10. Sloot R, Schim van der Loeff MF, Kouw PM, et al. Yield of tuberculosis contact investigations in Amsterdam: opportunities for improvement. Eur Respir J 2014;44:714-24.

11. Reichler MR, Reves R, Bur S, et al. Treatment of latent tuberculosis infection in contacts of new tuberculosis cases in the United States. South Med J 2002;95:414-20.

12. Marks SM, Taylor Z, Qualls NL, et al. Outcomes of contact investigations of infectious tuberculosis patients. Am J Respir Crit Care Med 2000;162:2033-8.

13. Sprinson JE, Flood J, Fan CS, et al. Evaluation of tuberculosis contact investigations in California. Int J Tuberc Lung Dis 2003;7:S363-8.

14. Jereb J, Etkind SC, Joglar OT, et al. Tuberculosis contact investigations: outcomes in selected areas of the United States, 1999. Int J Tuberc Lung Dis 2003;7:S384-90.

15. Aisu T, Raviglione MC, van Praag E, et al. Preventive chemotherapy for HIV-associated tuberculosis in Uganda: an operational assessment at a voluntary counselling and testing centre. AIDS 1995;9:267-74.

16. Chakhaia T, Magee MJ, Kempker RR, et al. High utility of contact investigation for latent and active tuberculosis case detection among the contacts: a retrospective cohort study in Tbilisi, Georgia, 20102011. PLoS One 2014;9:e111773. 
17. Rutherford ME, Ruslami R, Anselmo M, et al. Management of children exposed to Mycobacterium tuberculosis: a public health evaluation in West Java, Indonesia. Bull World Health Organ 2013:91:932-41.

18. Lopetegui M, Yen PY, Lai A, et al. Time motion studies in healthcare: what are we talking about? J Biomed Inform 2014;49:292-9.

19. Uwimana J, Zarowsky $\mathrm{C}$, Hausler $\mathrm{H}$, et al. Training community care workers to provide comprehensive TB/HIV/PMTCT integrated care in KwaZulu-Natal: lessons learnt. Trop Med Int Health 2012:17:488-96.

20. Talukder K, Salim MA, Jerin I, et al. Intervention to increase detection of childhood tuberculosis in Bangladesh. Int J Tuberc Lung Dis 2012;16:70-5.

21. Duarte R, Neto M, Carvalho A, et al. Improving tuberculosis contact tracing: the role of evaluations in the home and workplace. Int $J$ Tuberc Lung Dis 2012;16:55-9.

22. Cheng TL, Ottolini MC, Baumhaft K, et al. Strategies to increase adherence with tuberculosis test reading in a high-risk population. Pediatrics 1997;100:210-3.

23. Günal MM, Pidd M. Discrete event simulation for performance modelling in health care: a review of the literature. J Simul 2010;4:42-51.

24. Breslow NE. Cohort analysis in epidemiology. New York, NY: Springer, 1985:109-43.

25. Scott JM, deCamp A, Juraska M, et al. Finite-sample corrected generalized estimating equation of population average treatment effects in stepped wedge cluster randomized trials. Stat Methods Med Res 2017;26:583-97.

26. Demidenko E. International Statistical Review. 2007;75:96-113.

27. Dean CB, Lundy ER. Overdispersion. Wiley StatsRef: Statistics Reference Online, 2016:1-9.

28. Fox GJ, Barry SE, Britton WJ, et al. Contact investigation for tuberculosis: a systematic review and meta-analysis. Eur Respir $J$ 2013;41:140-56

29. Hirsch-Moverman Y, Daftary A, Franks J, et al. Adherence to treatment for latent tuberculosis infection: systematic review of studies in the US and Canada. Int J Tuberc Lung Dis 2008;12:1235-54.

30. Munro SA, Lewin SA, Smith HJ, et al. Patient adherence to tuberculosis treatment: a systematic review of qualitative research. PLoS Med 2007:4:e238.

31. Dumont A, Fournier P, Abrahamowicz M, et al. Quality of care, risk management, and technology in obstetrics to reduce hospital-based maternal mortality in Senegal and Mali (QUARITE): a clusterrandomised trial. Lancet 2013;382:146-57.

32. Manandhar DS, Osrin D, Shrestha BP, et al. Effect of a participatory intervention with women's groups on birth outcomes in Nepal: cluster-randomised controlled trial. Lancet 2004;364:970-9.

33. Morrison J, Pai M, Hopewell PC. Tuberculosis and latent tuberculosis infection in close contacts of people with pulmonary tuberculosis in low-income and middle-income countries: a systematic review and meta-analysis. Lancet Infect Dis 2008;8:359-68. 